important. Another important advantage of the oscillator approach over the sum over states approach is that in the latter calculation there are important interferences and cancellations among large terms (3). This makes it hard to develop physical intuition, and the computation becomes very sensitive to approximations such as truncation. Also in a sum over states approach, it is impossible to identify beforehand which states will be dominant. The search for the "essential states" is a major difficulty in the modeling of optical nonlinearities. In the oscillator approach, only the relevant oscillators show up in the equations of motion. We thus do not end up calculating a large number of unnecessary quantities.

It should be emphasized that there is no simple one to one correspondence between oscillators and individual eigenstates. The oscillators are collective excitations, and each oscillator represents many states. The precise relation between the two pictures is a highly nontrivial question that needs to be explored further. Symmetry plays a very different role in the eigenstate and in the oscillator representation. In the present model, the Hamiltonian is block diagonal into $A_{g}$ and $B_{u}$ symmetry parts, and all of the oscillators may be classified into either $A_{g}$ or $B_{u}$ type. In contrast to the description in terms of eigenstates of the Hamiltonian, where $B_{u}$ and $A_{g}$ states do not couple at all, $B_{u}$ and $A_{g}$ oscillators do couple in the equation of motion (Eq. 13). This fundamental difference between the oscillator and the eigenstate expansions is related to the nonlinear form of the equations of motion (a product of $B_{u}$ and $A_{g}$ variables can have a $B_{u}$ character), as opposed to the eigenstates expansion which are linear. Potentially this allows for a relatively inexpensive method for describing complex physical situations, compared with the expenditure required for eigenstate expansions.

With the current representation, we can use the same language to describe very different materials and answer some of the pressing questions raised recently (6). In particular, we can draw a close analogy between conjugated polyenes and semiconductor quantum dots (9). The physical picture of geometrically confined electron-hole pairs shows an origin in common with the blue shift of linear absorption as the size is decreased, and with the scaling and saturation of optical nonlinearities.

\section{REFERENCES AND NOTES}

1. J. F. Heflin, K. Y. Wong, Q. Zamani-Khamiri, A. F. Garito, Phys. Rev. B 38, 157 (1988); W. E. Torruellas D. Neher, R. Zanoni, G. I. Stegeman, F. Kajzar, Chem. Phys. Lett. 175, 11 (1990); M. Cha et al., ibid., in press.

2. S. Etemad and Z. G. Soos, in Spectroscopy of Ad vanced Materials, R. J. H. Clark and R. E. Hester,
Eds. (Wiley, New York, 1991), pp. 87-133, and ref erences therein

3. S. Mukamel, in Molecular Nonlinear Optics, J. Zyss, Ed. (Academic Press, New York, 1994), pp. 1-46 Principles of Nonlinear Optical Spectroscopy (Oxford, Univ. Press, New York, in press).

4. W. J. Buma, B. E. Kohler, T. A. Schuler, J. Chem Phys. 96, 399 (1992)

5. S. R. Marder, D. N. Beratan, L.-T. Cheng, Science 252, 103 (1991); S. R. Marder et al., ibid. 261, 186 (1993); S. M. Risser, D. N. Beratan, S. R. Marder, J. Am. Chem. Soc. 115, 7719 (1993).

6. B. I. Greene, J. Orenstein, S. Schmitt-Rink, Science 247, 679 (1990)

7. J. L. Bredas, C. Adant, P. Tackx, A. Persoons, B. M. Pierce, Chem. Rev. 94, 243 (1994).

8. C. Flytzanis and J. Hutter, in Contemporary Nonlinear Optics, G. P. Agrawal and R. W. Boyd, Eds. (Academic Press, San Diego, 1992), pp. 297-365.
9. L. Banyai and S. W. Koch, Semiconductor Quantum Dots (World Scientific, New York, 1993).

10. P. Fulde, Electron Correlations in Molecules and Solids (Springer, New York, 1993).

11. H. Fukutome, J. Mol. Struct. (Theochem.) 188, 337 (1989), and references therein

12. N. Bloembergen, Nonlinear Optics (Benjamin, New York, 1965)

13. A. Takahashi and S. Mukamel, J. Chem. Phys. 100 2366 (1994)

14. I. Ohmine, M. Karplus, K. Schulten, ibid. 68, 2298 (1978).

15. This research was supported by the Air Force Office of Scientific Research, The National Science Foundation (NSF), and the NSF Center for Photoinduced Charge Transfer.

1 June 1994; accepted 17 August 1994

\title{
Simulating the Self-Assembly of Gemini (Dimeric) Surfactants
}

\author{
S. Karaborni, ${ }^{*}$ K. Esselink, P. A. J. Hilbers, B. Smit, \\ J. Karthäuser, N. M. van Os, R. Zana
}

The morphologies and dynamics of aggregates formed by surfactant molecules are known to influence strongly performance properties spanning biology, household cleaning, and soil cleanup. Molecular dynamics simulations were used to investigate the morphology and dynamics of a class of surfactants, the gemini or dimeric surfactants, that are of potential importance in several industrial applications. Simulation results show that these surfactants form structures and have dynamic properties that are drastically different from those of single-chain surfactants. At the same weight fraction, single-chain surfactants form spherical micelles whereas gemini surfactants, whose two head groups are coupled by a short hydrophobic spacer, form thread-like micelles. Simulations at different surfactant concentrations indicate the formation of various structures, suggesting an alternative explanation for the unexpected viscosity behavior of gemini surfactants.

It is well known that surfactant molecules, which contain a hydrophobic chain and a hydrophilic head group, can form a variety of aggregates with properties different from those of the unassembled molecules. Indeed, spherical micelles, rod-like micelles, bilayers, reverse micelles, and vesicles have all been observed. This polymorphism forms the basis of many biochemical processes and is used in many industrial and household applications. A detailed understanding of surfactant selfassembly is therefore important in processes ranging from the transport of molecules through a cell membrane to the removal of stains in a washing machine (1).

In 1974 , a class of surfactants was synthesized; these surfactants have two hydrophobic chains and two hydrophilic head groups connected with a spacer (2). These surfactants, which were later termed gemini (3) or dimeric surfactants (4), have unusual char-

S. Karaborni, K. Esselink, P. A. J. Hilbers, B. Smit, J. Karthäuser, N. M. van Os, Shell Research B.V., Koninklijke/Shell-Laboratorium, Amsterdam, Post Office Box 38000, 1030 BN Amsterdam, Netherlands.

R. Zana, Institut Charles Sadron, Centre National de la Recherche Scientifique, 6 rue Boussingault, 67083 Strasbourg Cedex, France.

*To whom correspondence should be addressed. acteristics, such as a very low critical micelle concentration and a high efficiency in reducing the oil-water interfacial tension in comparison with conventional single-chain surfactants. These properties suggest that gemini may be excellent surfactants for soil cleanup and enhanced oil recovery; gemini are also possible candidates for the next generation of surfactants (5).

In a mixture of water and single-chain surfactants, the system tries to minimize its free energy by forming micelles in which the hydrophobic chains are brought together to minimize the contact with water. The hydrophilic head groups are kept near the water and away from each other as a result of electrostatic repulsions. In a gemini surfactant the two head groups are chemically linked by a hydrophobic spacer; as a consequence, a compromise has to be found for the location of the spacer depending on its length and flexibility and on the degree of repulsion between the two similarly charged head groups. For example, when the spacer length is shorter than the equilibrium distance between two charged head groups, the spacer will be fully extended to minimize the repulsion between the head groups. This positioning leads to a large unfavorable contact 
of the spacer with the water. In cases where the spacer is longer than the equilibrium distance between two charged head groups, the spacer will not be fully extended and will try to minimize its contact with the water. This reaction will, of course, depend to a large extent on the flexibility of the spacer. Therefore, characteristics such as the spacer's length and flexibility are essential in determining the shape of the surfactant aggregate.

To investigate systematically the effect of the spacer characteristics such as length and flexibility, we have performed molecular dynamics simulations on a model water-surfactant system. This model's main features are the preferred interactions of the hydrophilic surfactant head groups with the solvent and the preferred interactions of the hydrophobic chain groups with each other. With this simple model, we were able to predict that single-chain surfactants with different head groups would self-assemble into various shaped micelles $(6,7)$. The shapes of the sembled those found experimentally $(8,9)$ [and predicted by geometric principles (10)]. In addition to successfully predicting the geometry of single-chain surfactant aggregates, the simple model had been used to predict the operation of three mechanisms for oil solubilization in micellar solutions and to calculate the rates of solubilization (11).

The five molecules shown in Fig. 1 have been investigated in these simulations: four dimeric surfactants with spacers of various lengths and a single tail surfactant. In all simulations, the initial positions of the surfactant molecules were randomly chosen and the weight fractions were equal $(\approx 16 \%)$.

Figure 2 shows the structures that are formed by self-assembly. The differences among these structures are striking. In general, the geometries of aggregates formed by gemini surfactants are very different from micelles found in the simulations closely re-

those formed by single-chain surfactants. Whereas the single-chain surfactants form mostly spherical micelles (see Fig. 2A), gemini surfactants with a single spacer (spacer $=1$ ) form linear thread-like micelles (Fig. 2B), and gemini surfactants with a spacer of two or more oil-like particles $($ spacer $=2$ ) form a mixture of spheroids and tree-like micelles (Fig. 2C). Our results for the short spacer (spacer $=1$ ) are in agreement with the cryo-transmission electron micrographs of Zana and Talmon (4), which show that dimerization of $n$-dodecyltrimethylammonium bromide (DTAB) to a $12-2-12,2 \mathrm{Br}^{-}$ bis (quaternary ammonium bromide) molecule in which the two dodecyl chains $\left(\mathrm{C}_{12}\right)$ are linked at the head groups by a $-\left(\mathrm{CH}_{2}\right)_{2}-$ spacer $\left(\mathrm{C}_{2}\right)$ gives strongly entangled threadlike micelles.

The formation of a specific aggregate shape for a certain gemini surfactant depends on the equilibrium separation between head groups due to repulsions, the degree of hydrophobicity of the spacer (that is, its tendency to be away from the solvent), and the amount of flexibility of the spacer (that is, its capability to pack away from the solvent). It is foreseeable that at different spacer lengths, and thus spacer locations, aggregates with different shapes will form.

One of the surprising properties of gemini surfactants has been observed by Kern and colleagues (12). They report that the viscosity of a solution, consisting of a gemini surfactant having a short spacer, increases six orders of magnitude as the surfactant concentration is increased. This increase in viscosity is attributed to the formation of a network of entangled thread-like micelles. Upon further increase in concentration, Kern et al. noted an unexpected decrease in viscosity, a fact that they attributed to the decrease in effective length of the threadlike micelles, which in turn is due to a

Fig. 1. Various molecules used in simulations. Water molecules are represented by one water-like particle. Surfactants are represented by a combination of water-like and oil-like particles. Water-like particles interact by way of a Lennard-Jones potential with a cutoff chosen to be large enough to include excluded volume effects and attractive forces. Surfactant tail particles interact with each other by way of a Lennard-Jones potential with a large cutoff and with the water molecules by way of a LennardJones potential with a cutoff small enough to include no attractive forces. Surfactant head groups interact with each other through the repulsive part (excluded volume) of the Lennard-Jones potential and with the water by way of a Lennard-Jones potential with a large cutoff (water-like particles) (7). Particles

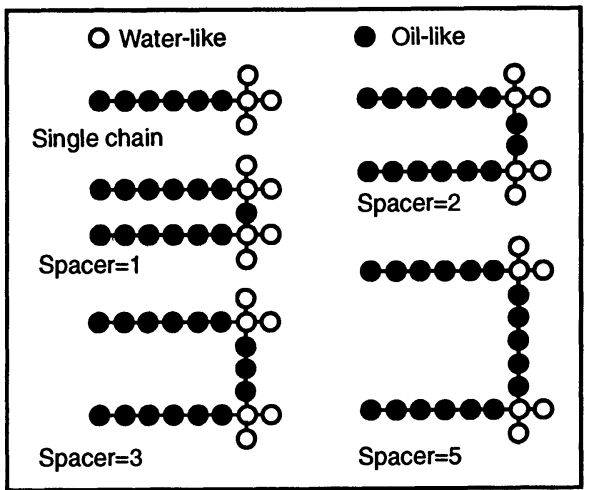

on the surfactant tails and the spacers do not correspond to a single methylene or methyl group but to approximately two to three methylenes. Neighboring particles on the same molecule are connected by harmonic springs of length $\sigma$, the size parameter in the Lennard-Jones potential. The simulations, which were done on a 400 transputer parallel computer, were performed at constant number of particles $(N)$, volume $(V)$, and reduced temperature, $k T / \epsilon=2.4$, where $\epsilon$ is the energy parameter in the Lennard-Jones potential. The simulation box consisted of 32,000 particles periodically repeated in all three directions.
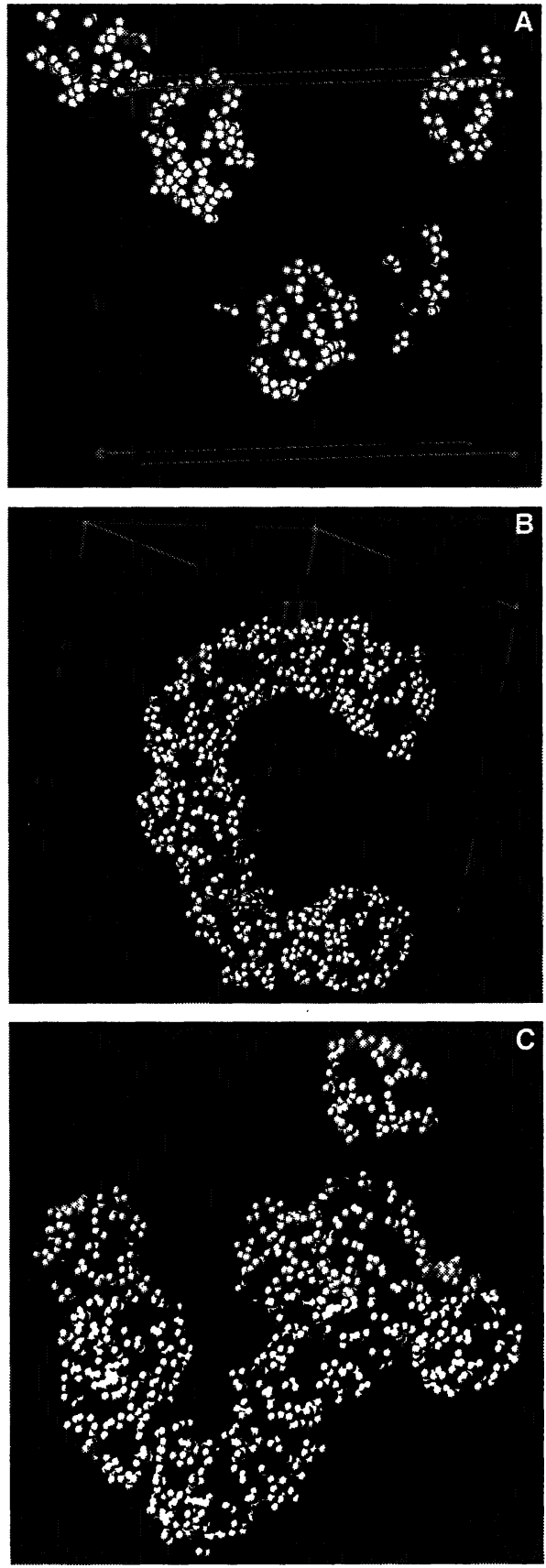

Fig. 2. (A) Representative snapshot of the simulation box of the single-chain surfactant shown in Fig. 1. At the end of the simulation there were 19 micelles formed. The most probable aggregate number is 23 . For reasons of clarity, only a few aggregates chosen randomly are shown in the snapshot. (B) Instantaneous configuration of one aggregate containing 189 gemini surfactants with a spacer of one oil-like particle between the head groups (spacer = 1 in Fig. 1). (C) Instantaneous configuration of two aggregates of 249 and 18 gemini surfactants with a spacer of two oil-like particles between the head groups (spacer $=2$ in Fig. 1). In all three simulations, the weight fraction of surfactants is $\approx 16 \%$. For reasons of clarity, water molecules are not shown, the particles that belong to the head groups are displayed in yellow, and those belonging to the surfactant tails are shown in red. 
decrease in electrostatic interactions (12). In our simulations, we observed that at low concentrations the gemini surfactants with the short spacer ( pacer $=1$ ) formed linear thread-like micelles (Fig. 2B). At higher concentrations, tree-like micelles were formed with random connections between the trunk and branches (Fig. 3).

Such a drastic change in aggregate structure usually has a significant influence on viscosity. Ideally, one would be able to calculate the viscosity directly from our simulations. Because the viscosity is extremely sensitive to the entanglements of various micelles and only very few micelles are formed in the present gemini simulations, such a calculation would suffer from finite size effects. To make the connection between the observed changes in the structures of the aggregates and the viscosity behavior, we rely on the theory of Lequeux (13). In this theory, a reptation model is used to show that branched micelles with sliding crosslinks have a lower viscosity than the corresponding thread-like micelles, suggesting that the decrease of viscosity observed by Kern et al. (12) may be due to the formation of tree-like micelles.

There is no experimental evidence that tree-like micelles exist. In view of the novelty of these self-assembled shapes, it is worthwhile to understand how they are formed. In our simulations, we observed that tree-like micelles formed in different stages. At the start of the simulation there were only monomeric gemini that aggregated initially into spherical micelles. These micelles subsequently collided to form cylinders. Both of these processes seem to be limited to a large extent by diffusion, either of the monomers or of spherical micelles. However, when cylinders are formed, several collisions are needed before a successful aggregation is seen (Fig. 4A). Successful collisions appear

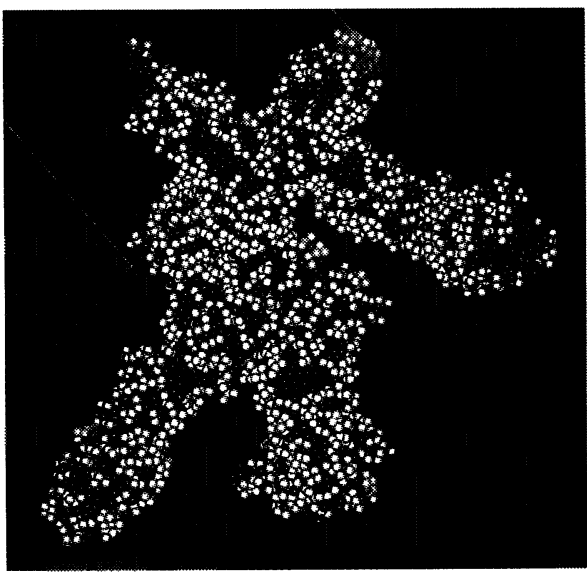

Fig. 3. Instantaneous configuration of one aggregate formed by gemini surfactants with a spacer of one oil-like particle between the head groups (spacer $=1$ in Fig. 1). Surfactant weight fraction is $\approx 28 \%$. Colors are the same as those in Fig. 2 . to occur when a section on the surface of the aggregate that has a low density of hydrophilic head groups of the surfactants comes into contact with a similar section from a different aggregate.

Collisions between cylinders sometimes lead to randomly branched structures, even though, from an energetic point of view, "reactions" between the cylinders' caps are more favorable and more probable. In fact, tree-like micelles have been theoretically judged to be unlikely because of the presence of "seams." The cost in bending energy of these "seams" is assumed to be prohibitive because the surfactant films are bent on a molecular scale (14). Theories of surfactant self-assembly stipulate that aggregates have a preferred curvature and molecular packing that are determined by the molecular struc-
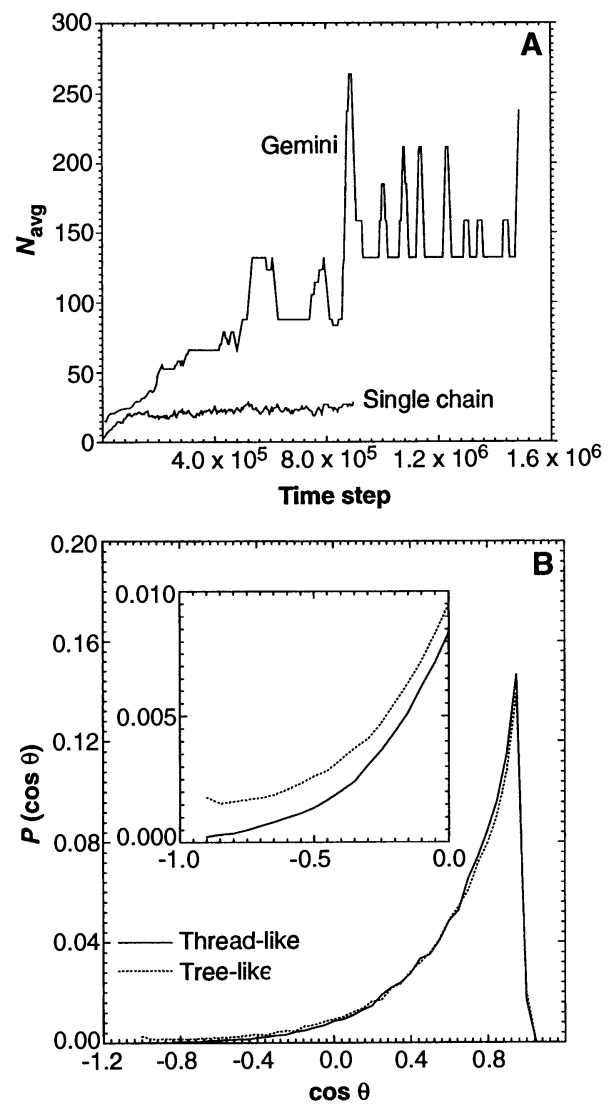

Fig. 4. (A) Running average aggregate size as a function of simulation time steps. The average aggregation size for single-chain surfactants reaches equilibrium more quickly than the average size for gemini surfactants. The variation is due to the different growing mechanisms for aggregates consisting of single-chain and gemini surfactants. (B) Probability of finding an angle $\theta$ between the two chains of the gemini surfactant with spacer $=1$. At $\cos \theta=1$, the two chains are parallel to each other; at $\cos \theta=0$, the two chains are perpendicular to each other; and at $\cos \theta=-1$, the two chains are pointing in opposite directions. The inset shows the probability only for those molecules in which the two chains form an angle of $90^{\circ}$ or more with each other. ture of the surfactants (10). Thus, aggregates, which assume a curvature or packing drastically different from the preferred curvature or packing, have a high bending energy and so have a low probability of being present in solution. The formation of tree-like micelles indicates the coexistence of a molecular packing in the "branches" and "trunks" that is drastically different from that in the "seams."

Tree-like micelles are likely characteristic of aggregates formed by gemini surfactants because of the presence of two tails and a chemical link between the two head groups of the gemini. One way to test this conclusion is to compare the orientation of molecules in the thread-like and tree-like micelles. The results in both cases show that the two chains of the gemini surfactant tend to be parallel to each other (Fig. 4B). This orientation is expected because the number of gemini in the "trunks" and "branches" of the tree-like micelle significantly outnumber the surfactants in the "seams." However, if one looks only at the molecules for which the chains make an angle of $90^{\circ}$ or more with each other, it is clear that the probability of such molecules is consistently higher in the tree-like than in the thread-like micelles. This difference is due to those molecules that are located in the "seams" of treelike micelles. Molecules in which the two tails form an angle of $90^{\circ}$ or more with each other are capable of lying partly in the "branch" and partly in the "trunk" of the tree-like micelles. These molecules act as locks between the "branch" and "trunk," overcoming the high cost in free energy associated with the nonpreferred curvature and molecular packing.

\section{REFERENCES AND NOTES}

1. H. Hoffmann and A. Stürmer, Tenside Surf. Deterg 30, 335 (1993)

2. Y. Deinega, Z. R. Ul'berg, L. G. Marochko, V. P. Rudi, V. P. Denisenko, Kolloidn. Zh. 36, 649 (1974)

3. F. M. Menger and C. A. Littau, J. Am. Chem. Soc. 113, 1451 (1991)

4. R. Zana and Y. Talmon, Nature 362, 228 (1993).

5. M. J. Rosen, Chemtech 23, 30 (1993).

6. B. Smit et al., Nature 348, 624 (1990).

7. B. Smit et al., Langmuir 9, 9 (1993).

8. B. Cabane, R. Duplessix, T. Zemb, J. Phys. (Paris) 46, 2161 (1985)

9. P. Vinson and Y. Talmon, J. Colloid Interface Sci. 139,288 (1989)

10. J. N. Israelachvili, D. J. Mitchell, B. W. Ninham, J. Chem. Soc., Faraday Trans. 2 72, 1525 (1976); J. N. Israelachvili, Intermolecular and Surface Forces (Academic Press, London, ed. 2, 1992), pp. 366-389.

11. S. Karaborni, N. M. van Os, K. Esselink, P. A. J. Hilbers, Langmuir 9, 1175 (1993)

12. F. Kern, F. Lequeux, R. Zana, S. J. Candau, ibid. 10 1714 (1994).

13. F. Lequeux, Europhys. Lett. 19, 675 (1992).

14. M. E. Cates and S. J. Candau, J. Phys. Condens. Matter 2, 6869 (1990); D. Roux, C. Coulon, M. E. Cates, J. Phys. Chem. 96, 4174 (1992); M. S. Turner, C. Marques, M. E. Cates, Langmuir 9, 695 (1993).

15. We thank A. van Helden and D. Frenkel for helpfu comments on the manuscript, and we are grateful to the Shell group for allowing the publication of these results.

20 June 1994; accepted 4 August 1994 